\title{
Discrete Gamma (Factorial) Function and Its Series in Terms of a Generalized Difference Operator
}

\author{
G. Britto Antony Xavier, V. Chandrasekar, \\ S. U. Vasanthakumar, and B. Govindan \\ Department of Mathematics, Sacred Heart College, Tirupattur 635601, India \\ Correspondence should be addressed to G. Britto Antony Xavier, shcbritto@yahoo.co.in
}

Received 17 July 2012; Accepted 5 October 2012

Academic Editor: Rüdiger Weiner

Copyright (c) 2012 G. Britto Antony Xavier et al. This is an open access article distributed under the Creative Commons Attribution License, which permits unrestricted use, distribution, and reproduction in any medium, provided the original work is properly cited.

The recent theory and applications of difference operator $\Delta_{L}$ introduced in (M. Maria Susai Manuel et al., 2012) are enriched and extended with a useful tool $\Delta_{\ell}^{-m}\left[k_{\ell}^{(n)} e^{-k}\right] \|_{(m-1) \ell+j}^{k}$ for finding the values of various series of discrete gamma functions in number theory. Illustrative examples show the effectiveness of the obtained results in finding the values of various gamma series.

\section{Introduction}

The fractional calculus involving gamma function is a generalization of differential calculus, allowing to define derivatives of real or complex order [1,2]. It is a mathematical subject that has proved to be very useful in applied fields such as economics, engineering, and physics [3-7]. In 1989, Miller and Ross introduced the discrete analogue of the Riemann-Liouville fractional derivative and proved some properties of the fractional difference operator [8]. In the general fractional $h$-difference Riemann-Liouville operator mentioned in $[9,10]$, the presence of the $h$ parameter is particularly interesting from the numerical point of view, because when $h$ tends to zero the solutions of the fractional difference equations can be seen as approximations to the solutions of corresponding Riemann-Liouville fractional differential equation $[9,11]$. On the other hand, fractional $h$ sum of order $m \geq 1\left(\left(\Delta_{h}^{-m} f\right)(t)\right.$ Definition 2.8 of [9]) is very useful to derive many interesting results in a different way in number theory such as the sum of the $m$ th partial sums on $n$th powers of arithmetic, arithmetic-geometric progressions, and products of $n$ consecutive terms of arithmetic progression using $\Delta_{\ell}^{-m} u(k)$ [12].

We observed that no results in number theory using definition 2.8 of [9] had been derived. In this paper, we use Definition 2.8 of [9] in a different way and define discrete gamma factorial function to obtain summation formulas of certain series on gamma function 
and gamma factorial function in number theory by getting closed and summation form of $\Delta_{\ell}^{-m} u(k) \|_{(m-1) \ell+j^{\prime}}^{k}$ (here we replace $f(t)$ by $u(k), h$ by $\ell$, and $v$ by $m$ on the notations used in $[9])$.

\section{Preliminaries}

Before stating and proving our results, we present some notations, basic definitions, and preliminary results which will be useful for further subsequent discussions. Let $\ell>0, k \in$ $[0, \infty), j=k-[k / \ell] \ell$ where $[k / \ell]$ denotes the integer part of $k / \ell, \mathbb{N}_{\ell}(j)=\{j, \ell+j, 2 \ell+j, \ldots\}$ and $\mathbb{N}_{1}(j)=\mathbb{N}(j)$. Throughout this paper, $c_{j}$ is a constant for all $k \in \mathbb{N}_{\ell}(j)$ and for any positive integer $m$, we denote $\left.\Delta_{\ell}^{-m} u(k)\right|_{(m-1) \ell+j} ^{k}=\left.\Delta_{\ell}^{-1}\left(\left.\cdots \Delta_{\ell}^{-1}\left(\left.\Delta_{\ell}^{-1} u(k)\right|_{j} ^{k}\right)\right|_{\ell+j} ^{k} \cdots\right)\right|_{(m-1) \ell+j}{ }^{k}$ where $\left.\Delta_{\ell}^{-1} u(k)\right|_{j} ^{k}=u_{1}(k)=\Delta_{\ell}^{-1} u(k)-\Delta_{\ell}^{-1} u(j),\left.\Delta_{\ell}^{-1}\left(\left.\Delta_{\ell}^{-1} u(k)\right|_{j} ^{k}\right)\right|_{\ell+j} ^{k}=u_{2}(k)=\Delta_{\ell}^{-1} u_{1}(k)-\Delta_{\ell}^{-1} u_{1}(\ell+$ $j)$, and so on.

Definition 2.1 (see [13]). For a real valued function $u(k)$, the generalized difference operator $\Delta_{\ell}$ and its inverse are, respectively, defined as

$$
\begin{gathered}
\Delta_{\ell} u(k)=u(k+\ell)-u(k), \quad k \in[0, \infty), \ell \in(0, \infty), \\
\text { if } \Delta_{\ell} v(k)=u(k), \quad \text { then } v(k)=\Delta_{\ell}^{-1} u(k)+c_{j} .
\end{gathered}
$$

Definition 2.2 (see [10]). For $k, n \in(0, \infty)$, the $\ell$-factorial function is defined by

$$
k_{\ell}^{(n)}=\ell^{n} \frac{\Gamma(k / \ell+1)}{\Gamma(k / \ell+1-n)},
$$

where $\Gamma$ is the Euler gamma function and $k_{1}^{(n)}=k^{(n)}$.

Remark 2.3. When $n \in \mathbb{N}(1),(2.3)$, and its $\Delta_{\ell}$ difference become

$$
k_{\ell}^{(n)}=\prod_{t=0}^{n-1}(k-t \ell), \quad \Delta_{\ell} k_{\ell}^{(n)}=(n \ell) k_{\ell}^{(n-1)}
$$

Lemma 2.4 (see [13]). Let $S_{r}^{n \prime}$ s be the Stirling numbers of second kind. Then,

$$
k^{n}=\sum_{r=1}^{n} S_{r}^{n} \ell^{n-r} k_{\ell}^{(r)}
$$

Theorem 2.5 (see [13]). Let $u(k), k \in[0, \infty)$ be real valued function. Then for $k \in[\ell, \infty)$,

$$
\left.\Delta_{\ell}^{-1} u(k)\right|_{j} ^{k}=\sum_{r=1}^{[k / \ell]} u(k-r \ell) .
$$


Lemma 2.6. Let $v(k)$ and $w(k)$ be two real valued functions. Then,

$$
\Delta_{\ell}^{-1}[v(k) w(k)]=v(k) \Delta_{\ell}^{-1} w(k)-\Delta_{\ell}^{-1}\left[\Delta_{\ell}^{-1} w(k+\ell) \Delta_{\ell} v(k)\right] .
$$

Proof. From (2.1), we find

$$
\Delta_{\ell}[v(k) z(k)]=z(k+\ell) \Delta_{\ell} v(k)+v(k) \Delta_{\ell} z(k) .
$$

Applying (2.2) in (2.8), we obtain

$$
\Delta_{\ell}^{-1}\left[v(k) \Delta_{\ell} z(k)\right]=v(k) z(k)-\Delta_{\ell}^{-1}\left[z(k+\ell) \Delta_{\ell} v(k)\right] .
$$

The proof follows by taking $w(k)=\Delta_{\ell} z(k)$ in (2.9).

\section{Main Results}

In this section, we use the following notations: $L_{m-1}=\{1,2, \ldots, m-1\}, 0\left(L_{m-1}\right)=\{\phi\}$, $\phi$ is an empty set, $1\left(L_{m-1}\right)=\{\{1\},\{2\}, \ldots,\{m-1\}\}, 2\left(L_{m-1}\right)=\{\{1,2\},\{1,3\}, \ldots,\{1, m-$ $1\},\{2,3\}, \ldots,\{2, m-1\}, \ldots,\{m-2, m-1\}\}$. In general, $t\left(L_{m-1}\right)=$ set of all subsets of size $t$ from the set $L_{m-1}$ such that if $\left\{m_{1}, m_{2}, \ldots, m_{t}\right\} \in t\left(L_{m-1}\right)$, then $m_{1}<m_{2}<\cdots<m_{t}$, $(m-1)\left(L_{m-1}\right)=\{\{1,2, \ldots, m-1\}\}, \wp\left(L_{m-1}\right)=\bigcup_{t=0}^{m-1} t\left(L_{m-1}\right)$, power set of $L_{m-1}, \sum_{t=1}^{m-1} f(t)=0$ for $m \leq 1$, and $\prod_{i=2}^{t} f(i)=1$ for $t \leq 1$.

Lemma 3.1 (see [13]). Let $k \in[\ell, \infty)$. Then,

$$
\left.\Delta_{\ell}^{-1} e^{-k}\right|_{j} ^{k}=\frac{e^{-k}}{e^{-\ell}-1}-\frac{e^{-j}}{e^{-\ell}-1}
$$

Lemma 3.2 (see [13]). Let $n$ be any nonnegative integer. Then,

$$
\left.\Delta_{\ell}^{-1} k_{\ell}^{(n)}\right|_{j} ^{k}=\frac{k_{\ell}^{(n+1)}}{\ell(n+1)}-\frac{j_{\ell}^{(n+1)}}{\ell(n+1)} .
$$

In particular, when $\ell=1, k=(r-1) \in \mathbb{N}=\{1,2,3, \ldots\}, j=0$, then (3.2) becomes

$$
\left.\Delta^{-1} k^{(n)}\right|_{0} ^{r-1}=\frac{(r-1)^{(n+1)}}{(n+1)}
$$

Remark 3.3. For any constant $c$, since $1=k_{\ell}^{(0)}$, by (3.2) and linearity of $\Delta_{\ell}^{-1}$,

$$
\left.\Delta_{\ell}^{-1} c\right|_{j} ^{k}=\left.c \Delta_{\ell}^{-1} k_{\ell}^{(0)}\right|_{j} ^{k}=c\left[\frac{k_{\ell}^{(1)}}{1 ! \ell}-\frac{j_{\ell}^{(1)}}{1 ! \ell}\right] .
$$


Lemma 3.4. Let $n \in \mathbb{N}(0)$ and $r \in \mathbb{N}(3)$. Then,

$$
\left.\Delta^{-1} k^{(n)}\right|_{0} ^{r-1}=(r-2)^{(n)}+(r-3)^{(n)}+\cdots+(1)^{(n)}=\frac{(r-1)^{(n+1)}}{(n+1)} .
$$

Proof. The proof follows by taking $\ell=1, u(k)=k^{(n)}$ in (2.6) and (3.2).

Theorem 3.5. Let $m$ be a positive integer, $\ell \in(0, \infty)$, and $k \in[m \ell, \infty)$. Then,

$$
\Delta_{\ell}^{-m} u(k) \|_{(m-1) \ell+j}^{k}=\sum_{r=m}^{[k / \ell]} \frac{(r-1)^{(m-1)}}{(m-1) !} u(k-r \ell) .
$$

Proof. Taking $\Delta_{\ell}^{-1}$ on (2.6), and applying (2.6) for $\Delta_{\ell}^{-1} u(k-r \ell)$, we get

$$
\left.\Delta_{\ell}^{-1}\left(\left.\Delta_{\ell}^{-1} u(k)\right|_{j} ^{k}\right)\right|_{\ell+j} ^{k}=\sum_{r=1}^{[k / \ell]} \Delta_{\ell}^{-1} u(k-r \ell)=\sum_{r=1}^{[k / \ell][(k-r \ell) / \ell]} \sum_{s=1} u(k-r \ell-s \ell)
$$

From the notation given this section and ordering the terms $u(k-r \ell)$, we find

$$
\Delta_{\ell}^{-2} u(k) \|_{\ell+j}^{k}=\sum_{r=2}^{[k / \ell]} \frac{(r-1)^{(1)}}{1 !} u(k-r \ell) .
$$

Again, taking $\Delta_{\ell}^{-1}$ on (3.8), by (2.6) for $\Delta_{\ell}^{-1} u(k-r \ell)$, we arrive

$$
\Delta_{\ell}^{-3} u(k) \|_{2 \ell+j}^{k}=\sum_{r=3}^{[k / \ell]}\left[\frac{(r-2)^{(1)}}{1 !}+\frac{(r-3)^{(1)}}{1 !}+\cdots+\frac{(1)^{(1)}}{1 !}\right] u(k-r \ell),
$$

which yields by (3.5),

$$
\Delta_{\ell}^{-3} u(k) \|_{2 \ell+j}^{k}=\sum_{r=3}^{[k / \ell]} \frac{(r-1)^{(2)}}{2 !} u(k-r \ell)
$$

Now, (3.6) will be obtained by continuing this process and using (3.5). 
Theorem 3.6. Let $m \in \mathbb{N}(2), \ell \in(0, \infty)$, and $k \in[m \ell, \infty)$. Then,

$$
\begin{aligned}
\Delta_{\ell}^{-m} u(k) \|_{(m-1) \ell+j}^{k}= & \left.\Delta_{\ell}^{-m} u(k)\right|_{(m-1) \ell+j} ^{k}+\sum_{t=1}^{m-1} \sum_{\left\{m_{1}, \ldots, m_{t}\right\} \in t\left(L_{m-1}\right)}(-1)^{t} \\
& \times\left(\Delta_{\ell}^{-m_{1}} u\left(\left(m_{1}-1\right) \ell+j\right)\right) \frac{k_{\ell}^{\left(m-m_{t}\right)}}{\left(m-m_{t}\right) ! \ell^{m-m_{t}}} \\
& \times\left.\prod_{i=2}^{t} \frac{\left(\left(m_{i}-1\right) \ell+j\right)_{\ell}^{\left(m_{i}-m_{i-1}\right)}}{\left(m_{i}-m_{i-1}\right) ! \ell^{m_{i}-m_{i-1}}}\right|_{(m-1) \ell+j} ^{k} .
\end{aligned}
$$

Proof. Applying the limit $j$ to $k$ on $\Delta_{\ell}^{-1} u(k)$, we write

$$
\left.\Delta_{\ell}^{-1} u(k)\right|_{j} ^{k}=\Delta_{\ell}^{-1} u(k)-\Delta_{\ell}^{-1} u(j)
$$

where $\Delta_{\ell}^{-1} u(j)$ is constant and $\Delta_{\ell}^{-1} u(k)$ is a function of $k$. Taking $\Delta_{\ell}^{-1}$ on (3.12), by (3.4) and applying the limit $\ell+j$ to $k$, we obtain

$$
\Delta_{\ell}^{-2} u(k) \|_{\ell+j}^{k}=\left.\Delta_{\ell}^{-2} u(k)\right|_{\ell+j} ^{k}-\left.\Delta_{\ell}^{-1} u(j) \frac{k_{\ell}^{(1)}}{1 ! \ell}\right|_{\ell+j} ^{k}
$$

which can be expressed as

$$
\Delta_{\ell}^{-2} u(k) \|_{\ell+j}^{k}=\left.\Delta_{\ell}^{-2} u(k)\right|_{\ell+j} ^{k}+\left.(-1)^{t} \Delta_{\ell}^{-m_{1}} u\left(\left(m_{1}-1\right) \ell+j\right) \frac{k_{\ell}^{\left(2-m_{t}\right)}}{\left(2-m_{t}\right) ! \ell^{2-m_{t}}}\right|_{\ell+j} ^{k}
$$

where $t=1$ and $\left\{m_{1}=m_{t}=1\right\} \in 1\left(L_{1}\right)$ and is same as

$$
\begin{aligned}
\Delta_{\ell}^{-2} u(k) \|_{\ell+j}^{k}= & \Delta_{\ell}^{-2} u(k)-\Delta_{\ell}^{-2} u(\ell+j)+\frac{(-1)^{t} k_{\ell}^{\left(2-m_{t}\right)}}{\left(2-m_{t}\right) ! \ell^{2-m_{t}}} \Delta_{\ell}^{-m_{1}} u\left(\left(m_{1}-1\right) \ell+j\right) \\
& -\frac{(-1)^{t}(\ell+j)_{\ell}^{\left(2-m_{t}\right)}}{\left(2-m_{t}\right) ! \ell^{2-m_{t}}} \Delta_{\ell}^{-m_{1}} u\left(\left(m_{1}-1\right) \ell+j\right),
\end{aligned}
$$

where all the terms except $\Delta_{\ell}^{-2} u(k), k_{\ell}^{\left(2-m_{t}\right)}$ are constants. 
Again taking $\Delta_{\ell}^{-1}$ on (3.15), by (3.2) and (3.4), we arrive

$$
\begin{aligned}
\Delta_{\ell}^{-3} u(k) \|_{2 \ell+j}^{k}= & \left.\Delta_{\ell}^{-3} u(k)\right|_{2 \ell+j} ^{k}-\left.\Delta_{\ell}^{-2} u(\ell+j) \frac{k_{\ell}^{(1)}}{1 ! \ell}\right|_{2 \ell+j} ^{k} \\
& +\left.(-1)^{1} \Delta_{\ell}^{-m_{1}} u\left(\left(m_{1}-1\right) \ell+j\right) \frac{k_{\ell}^{\left(3-m_{t}\right)}}{\left(3-m_{t}\right) ! \ell^{3-m_{t}}}\right|_{2 \ell+j} ^{k} \\
& +\left.(-1)^{2} \Delta_{\ell}^{-m_{1}} u\left(\left(m_{1}-1\right) \ell+j\right) \frac{k_{\ell}^{(1)}}{1 ! \ell} \frac{(\ell+j)_{\ell}^{\left(2-m_{t}\right)}}{\left(2-m_{t}\right) ! \ell^{2-m_{t}}}\right|_{2 \ell+j} ^{k},
\end{aligned}
$$

which is the same as

$$
\begin{aligned}
\Delta_{\ell}^{-3} u(k) \|_{2 \ell+j}^{k}= & \left.\Delta_{\ell}^{-3} u(k)\right|_{2 \ell+j} ^{k}+\sum_{t=1}^{2} \sum_{\substack{\left\{m_{t}=1\right\},\left\{m_{t}=2\right\} \in 1\left(L_{2}\right) \\
\left\{m_{1}=1, m_{t}=2\right\} \in 2\left(L_{2}\right)}}(-1)^{t} \Delta_{\ell}^{-m_{1}} u\left(\left(m_{1}-1\right) \ell+j\right) \\
& \times\left.\frac{k_{\ell}^{\left(3-m_{t}\right)}}{\left(3-m_{t}\right) ! \ell^{3-m_{t}}} \frac{\left(\left(m_{i}-1\right) \ell+j\right)_{\ell}^{\left(m_{i}-m_{i-1}\right)}}{\left(m_{i}-m_{i-1}\right) ! \ell^{m_{i}-m_{i-1}}}\right|_{2 \ell+j} ^{k},
\end{aligned}
$$

where $i=2$. In the same way, we find

$$
\begin{aligned}
\Delta_{\ell}^{-4} u(k) \|_{3 \ell+j}^{k}= & \left.\Delta_{\ell}^{-4} u(k)\right|_{3 \ell+j} ^{k}+\sum_{t=1}^{3} \sum_{\substack{\left\{m_{t}\right\} \in 1\left(L_{3}\right),\left\{m_{1}, m_{t}\right\} \in 2\left(L_{3}\right) \\
\left\{m_{1}, m_{2}, m_{t}\right\} \in 3\left(L_{3}\right)}}(-1)^{t} \Delta_{\ell}^{-m_{1}} u\left(\left(m_{1}-1\right) \ell+j\right) \\
& \times\left.\frac{k_{\ell}^{\left(4-m_{t}\right)}}{\left(4-m_{t}\right) ! \ell^{4-m_{t}}} \prod_{i=2}^{3} \frac{\left(\left(m_{i}-1\right) \ell+j\right)_{\ell}^{\left(m_{i}-m_{i-1}\right)}}{\left(m_{i}-m_{i-1}\right) ! \ell^{m_{i}-m_{i-1}}}\right|_{3 \ell+j} ^{k},
\end{aligned}
$$

which can be expressed as the following:

$$
\begin{aligned}
\Delta_{\ell}^{-4} u(k) \|_{3 \ell+j}^{k}= & \left.\Delta_{\ell}^{-4} u(k)\right|_{3 \ell+j} ^{k}+\sum_{t=1}^{3} \sum_{\left\{m_{1}, \ldots, m_{t}\right\} \in t\left(L_{3}\right)}(-1)^{t} \Delta_{\ell}^{-m_{1}} u\left(\left(m_{1}-1\right) \ell+j\right) \\
& \times\left.\frac{k_{\ell}^{\left(4-m_{t}\right)}}{\left(4-m_{t}\right) ! \ell^{4-m_{t}}} \prod_{i=2}^{3} \frac{\left(\left(m_{i}-1\right) \ell+j\right)_{\ell}^{\left(m_{i}-m_{i-1}\right)}}{\left(m_{i}-m_{i-1}\right) ! \ell^{m_{i}-m_{i-1}}}\right|_{3 \ell+j} ^{k} .
\end{aligned}
$$

The proof completes by continuing this process. 
Theorem 3.7 (partial summation formula). Let $m \in \mathbb{N}(2)$. Then,

$$
\begin{aligned}
\sum_{r=m}^{[k / \ell]} \frac{(r-1)^{(m-1)}}{(m-1) !} u(k-r \ell)= & \left.\Delta_{\ell}^{-m} u(k)\right|_{(m-1) \ell+j} ^{k}+\sum_{t=1}^{m-1} \sum_{\left\{m_{1}, \ldots, m_{t}\right\} \in t\left(L_{m-1}\right)}(-1)^{t} \\
& \times\left(\Delta_{\ell}^{-m_{1}} u\left(\left(m_{1}-1\right) \ell+j\right)\right) \frac{k_{\ell}^{\left(m-m_{t}\right)}}{\left(m-m_{t}\right) ! \ell^{m-m_{t}}} \\
& \times\left.\prod_{i=2}^{t} \frac{\left(\left(m_{i}-1\right) \ell+j\right)_{\ell}^{\left(m_{i}-m_{i-1}\right)}}{\left(m_{i}-m_{i-1}\right) ! \ell^{m_{i}-m_{i-1}}}\right|_{(m-1) \ell+j} ^{k} .
\end{aligned}
$$

Proof. The proof follows by equating (3.6) and (3.11).

Corollary 3.8. Let $m \in \mathbb{N}(2), \ell \in(0, \infty)$, and $k \in[m \ell, \infty)$. Then,

$$
\begin{aligned}
& \sum_{r=m}^{[k / \ell]} \frac{(r-1)^{(m-1)}}{(m-1) ! e^{(k-r \ell)}}(k-r \ell)_{\ell}^{(n)} \\
&=\sum_{r=1}^{n+1}(-1)^{r-1} \frac{n^{(r-1)}}{(r-1) !}\left[\frac{(m+r-2)^{(r-1)} \ell^{r-1}}{\left(e^{-\ell}-1\right)^{r+m-1}} \times \frac{k_{\ell}^{(n+1-r)}}{e^{(k+(r-1) \ell)}}+\sum_{t=1}^{m-1} \sum_{\left\{m_{1}, \ldots, m_{t}\right\} \in t\left(L_{m-1}\right)}\right. \\
& \times(-1)^{t} \frac{\left(m_{1}+r-2\right)^{(r-1)} \ell^{r-1}}{\left(e^{-\ell}-1\right)^{r+m_{1}-1}} \times \frac{\left(\left(m_{1}-1\right) \ell+j\right)_{\ell}^{(n+1-r)} k_{\ell}^{\left(m-m_{t}\right)}}{\left(m-m_{t}\right) ! \ell^{m-m_{t}} e^{\left(\left(m_{1}-1\right) \ell+j+(r-1) \ell\right)}} \\
&\left.\quad \times \prod_{i=2}^{t} \frac{\left(\left(m_{i}-1\right) \ell+j\right)_{\ell}^{\left(m_{i}-m_{i-1}\right)}}{\left(m_{i}-m_{i-1}\right) ! \ell^{m_{i}-m_{i-1}}}\right]_{(m-1) \ell+j}^{k} .
\end{aligned}
$$

Proof. Taking $v(k)=k_{\ell}^{(n)}, w(k)=e^{-k}, u(k)=v(k) w(k)$ in (2.7), by (2.4), (3.1), and (3.2), we get

$$
\Delta_{\ell}^{-1} u(k)=k_{\ell}^{(n)} \frac{e^{-k}}{e^{-\ell}-1}-\Delta_{\ell}^{-1}\left(\frac{e^{-k+\ell}}{e^{-\ell}-1}(n \ell) k_{\ell}^{(n-1)}\right) .
$$

By (3.1) and applying (2.7) for $k_{\ell}^{(n-1)} e^{-k}, k_{\ell}^{(n-2)} e^{-k}, \ldots, k_{\ell}^{(1)} e^{-k}$, we arrive

$$
\Delta_{\ell}^{-1} u(k)=\sum_{r=1}^{n+1}(-1)^{r-1} \frac{n^{(r-1)}}{(r-1) !}(r-1)^{(r-1)} \frac{\ell^{r-1}}{\left(e^{-\ell}-1\right)^{r}} \frac{k_{\ell}^{(n+1-r)}}{e^{(k+(r-1) \ell)}}
$$


Taking $\Delta_{\ell}^{-1}$ on and applying (3.23) for $(m-1)$ times, we arrive

$$
\Delta_{\ell}^{-m} u(k)=\sum_{r=1}^{n+1}(-1)^{r-1} \frac{n^{(r-1)}}{(r-1) !} \frac{(m+r-2)^{(r-1)} \ell^{r-1}}{\left(e^{-\ell}-1\right)^{r+m-1}} \frac{k_{\ell}^{(n+1-r)}}{e^{(k+(r-1) \ell)}} .
$$

The proof follows by taking $u(k)=k_{\ell}^{(n)} e^{-k}$ in Theorem 3.7.

The following example illustrates Corollary 3.8.

Example 3.9. Consider the case when $m=4$ and $n=2$. In this case, $L_{3}=\{1,2,3\}, 1\left(L_{3}\right)=$ $\{\{1\},\{2\},\{3\}\}, 2\left(L_{3}\right)=\{\{1,2\},\{1,3\},\{2,3\}\}, 3\left(L_{3}\right)=\{\{1,2,3\}\}$ and (3.21) becomes

$$
\begin{aligned}
\sum_{r=4}^{[k / \ell]} \frac{(r-1)^{(3)}(k-r \ell)_{\ell}^{(2)}}{(3) ! e^{(k-r \ell)}} & \begin{aligned}
& 3 \\
&=\sum_{r=1}^{3}(-1)^{r-1} \frac{2^{(r-1)}}{(r-1) !} \times {\left[\frac{(2+r)^{(r-1)} \ell^{r-1} k_{\ell}^{(1+r)}}{\left(e^{-\ell}-1\right)^{r+3} e^{(k+(r-1) \ell)}}+\sum_{t=1}^{3} \sum_{\left\{m_{1}, \ldots, m_{t}\right\} \in t\left(L_{3}\right)}(-1)^{t} \frac{\left(m_{1}+r-2\right)^{(r-1)} \ell^{r-1}}{\left(e^{-\ell}-1\right)^{r+m_{1}-1}}\right.} \\
&\left.\times \frac{\left(\left(m_{1}-1\right) \ell+j\right)_{\ell}^{(1+r)} k_{\ell}^{\left(4-m_{t}\right)}}{\left(4-m_{t}\right) ! \ell^{4-m_{t}} e^{\left(\left(m_{1}-1\right) \ell+j+(r-1) \ell\right)}} \prod_{i=2}^{t} \frac{\left(\left(m_{i}-1\right) \ell+j\right)_{\ell}^{\left(m_{i}-m_{i-1}\right)}}{\left(m_{i}-m_{i-1}\right) ! \ell^{m_{i}-m_{i-1}}}\right]_{3 \ell+j}^{k}
\end{aligned}
\end{aligned}
$$

The double summation expression of (3.25) will be obtained by adding the sums corresponds to $1\left(L_{3}\right)$ :

$$
\begin{aligned}
& \frac{(r-1)^{(r-1)} \ell^{r-1}}{\left(e^{-\ell-1}\right)^{r} e^{(j+(r-1) \ell)}}(j)_{\ell}^{(1+r)} \frac{k_{\ell}^{(3)}}{3 ! \ell^{3}}+\frac{(r)^{(r-1)} \ell^{r-1}}{\left(e^{-\ell-1}\right)^{r+1} e^{(\ell+j+(r-1) \ell)}}(\ell+j)_{\ell}^{(1+r)} \frac{k_{\ell}^{(2)}}{2 ! \ell^{2}} \\
& +\frac{(r+1)^{(r-1)} \ell^{r-1}}{\left(e^{-\ell-1}\right)^{r+2} e^{(2 \ell+j+(r-1) \ell)}}(2 \ell+j)_{\ell}^{(1+r)} \frac{k_{\ell}^{(1)}}{\ell}
\end{aligned}
$$

corresponds to $2\left(L_{3}\right)$ :

$$
\begin{aligned}
& \frac{(r-1)^{(r-1)} \ell^{r-1}}{\left(e^{-\ell-1}\right)^{r} e^{(j+(r-1) \ell)}}(j)_{\ell}^{(1+r)} \frac{k_{\ell}^{(2)}}{2 ! \ell^{2}} \frac{(\ell+j)_{\ell}^{(1)}}{\ell}+\frac{(r-1)^{(r-1)} \ell^{r-1}}{\left(e^{-\ell-1}\right)^{r} \mathrm{e}^{(j+(r-1) \ell)}}(j)_{\ell}^{(1+r)} \\
& \quad \times \frac{k_{\ell}^{(1)}}{\ell} \frac{(2 \ell+j)_{\ell}^{(2)}}{2 ! \ell^{2}}+\frac{(r)^{(r-1)} \ell^{r-1}}{\left(e^{-\ell-1}\right)^{r+1} e^{(\ell+j+(r-1) \ell)}}(\ell+j)_{\ell}^{(1+r)} \frac{k_{\ell}^{(1)}}{\ell} \frac{(2 \ell+j)_{\ell}^{(2)}}{2 ! \ell^{2}}
\end{aligned}
$$

and to $3\left(L_{3}\right)$ :

$$
\frac{(r-1)^{(r-1)} \ell^{r-1}}{\left(e^{-\ell-1}\right)^{r} e^{(j+(r-1) \ell)}}(j)_{\ell}^{(1+r)} \frac{k_{\ell}^{(1)}}{\ell} \frac{(\ell+j)_{\ell}^{(1)}}{\ell} \frac{(2 \ell+j)_{\ell}^{(1)}}{\ell} .
$$


Corollary 3.10. Let $\ell \in(0, \infty)$ and $k \in[\ell, \infty)$. Then,

$$
\left.\Delta_{\ell}^{-1}\left[k_{\ell}^{(n)} e^{-k}\right]\right|_{j} ^{k}=\sum_{r=1}^{[k / \ell]} \frac{(k-r \ell)_{\ell}^{(n)}}{e^{(k-r \ell)}}=\left.\sum_{r=1}^{n+1} \frac{-n^{(r-1)} \ell^{r-1} k_{\ell}^{(n+1-r)}}{\left(1-e^{-\ell}\right)^{r} e^{(k+(r-1) \ell)}}\right|_{j} ^{k}
$$

Proof. Since $k_{\ell}^{(n)} e^{-k}=0$ as $k \rightarrow \infty$ and $0_{\ell}^{(r)}=0$ if $r \neq 0$ and $0_{\ell}^{(r)}=1$ if $r=0$, the upper limit of (3.23) for $k \rightarrow \infty$ will be zero and lower limit for $j=0$, gives (3.29).

\section{Discrete Gamma-Factorial Function}

First we derive infinite series formula using $\Delta_{\ell}^{-1}$, which induces the definition of discrete gamma factorial function.

Theorem 4.1. Let $k \in[0, \infty)$ and $\lim _{k \rightarrow \infty} \Delta_{\ell}^{-1} u(k)=0$. Then,

$$
\left.\Delta_{\ell}^{-1} u(k)\right|_{k} ^{\infty}=\sum_{r=0}^{\infty} u(k+r \ell)
$$

Proof. From (2.6), and expressing its terms in reverse order, we find

$$
\left.\Delta_{\ell}^{-1} u(k)\right|_{j} ^{k}+\left.\Delta_{\ell}^{-1} u(k)\right|_{k} ^{\infty}=u(j)+u(\ell+j)+\cdots+u(k-\ell)+u(k)+u(k+\ell)+\cdots+u(\infty)
$$

which is the same as

$$
\left.\Delta_{\ell}^{-1} u(k)\right|_{j} ^{\infty}=\sum_{r=0}^{\infty} u(j+r \ell)
$$

Now (4.1) follows by given condition $\Delta_{\ell}^{-1} u(\infty)=0$ and then replacing $j$ by $k$.

Definition 4.2. For $n \in(-\infty, \infty)-\{0\}$ and $k \in[0, \infty)$, the discrete $k$-gamma factorial function is defined as

$$
{ }_{k} \Gamma_{\ell}((n))=\ell \Delta_{\ell}^{-1}\left[k_{\ell}^{(n-1)} e^{-k}\right]_{k}^{\infty}=\ell \sum_{r=0}^{\infty}(k+r \ell)_{\ell}^{(n-1)} e^{-(k+r \ell)},
$$

and the discrete $k$-gamma function is defined as

$$
{ }_{k} \Gamma_{\ell}(n)=\ell \Delta_{\ell}^{-1}\left[k^{n-1} e^{-k}\right]_{k}^{\infty}=\ell \sum_{r=0}^{\infty}(k+r \ell)^{n-1} e^{-(k+r \ell)}
$$


In particular, when $k=0,(4.4)$ and (4.5) becomes

$$
\begin{gathered}
\Gamma_{\ell}((n))=\ell \Delta_{\ell}^{-1}\left[k_{\ell}^{(n-1)} e^{-k}\right]_{k=0}^{\infty}=\ell \sum_{r=1}^{\infty}(r \ell)_{\ell}^{(n-1)} e^{-r \ell} \\
\Gamma_{\ell}(n)=\ell \Delta_{\ell}^{-1}\left[k^{n-1} e^{-k}\right]_{k=0}^{\infty}=\ell \sum_{r=1}^{\infty}(r \ell)^{n-1} e^{-r \ell}
\end{gathered}
$$

which can be called as the discrete gamma factorial function and the discrete gamma function, respectively.

Theorem 4.3. Let $\ell \in(0, \infty)$ and $n \in \mathbb{N}$. Then,

$$
{ }_{k} \Gamma_{\ell}((n+1))=\ell\left[\sum_{r=0}^{n} \frac{(n)^{(r)} \ell^{r} k_{\ell}^{(n-r)} e^{-(k+r \ell)}}{\left(1-e^{-\ell}\right)^{r+1}}\right]=\ell \sum_{r=0}^{\infty}(k+r \ell)_{\ell}^{(n)} e^{-(k+r \ell)} .
$$

Proof. The proof follows by taking $j=k, k=\infty$ in (3.29) and multiplying it by $\ell$.

Theorem 4.4. Let $\ell \in(0, \infty)$ and $n \in \mathbb{N}(1)$. Then,

$$
\Gamma_{\ell}((n+1))=\frac{\ell e^{-\ell}}{1-e^{-\ell}} n \Gamma_{\ell}((n))=n ! \frac{\ell^{n+1} e^{-n \ell}}{\left(1-e^{-\ell}\right)^{n+1}}
$$

Proof. From (2.7), (3.1), and (4.6), we get

$$
\Gamma_{\ell}((n+1))=\ell\left\{k_{\ell}^{(n)} \frac{e^{-k}}{e^{-\ell}-1}-\Delta_{\ell}^{-1}\left\{\frac{e^{-(k+\ell)}}{e^{-\ell}-1}(n \ell) k_{\ell}^{(n-1)}\right\}\right\}_{k=0}^{\infty}
$$

Since $k_{\ell}^{(n)} e^{-k}=0$ for $k=0$ and $k=\infty$, (4.10) gives first part of (4.9) by (4.6). Now second part of (4.9) will be obtained by applying first part of (4.9) again and again and using the identity $\Gamma_{\ell}((1))=\ell /\left(1-e^{-\ell}\right)$. 
Theorem 4.5. Let $S_{r}^{n}$ be as given in (2.5) and $n \in \mathbb{N}(1)$. Then,

$$
\Delta_{\ell}^{-m}\left[k^{n} e^{-k}\right]=\sum_{r=1}^{n} S_{r}^{n} \ell^{n-r} \Delta_{\ell}^{-m}\left[k_{\ell}^{(r)} e^{-k}\right]
$$

which gives

$$
\Gamma_{\ell}(n+1)=\sum_{r=1}^{n} S_{r}^{n} \ell^{n-r} \Gamma_{\ell}((r+1))=\frac{\ell e^{-\ell}}{1-e^{-\ell}} \sum_{r=1}^{n} S_{r}^{n} \ell^{n-r} r \Gamma_{\ell}((r))
$$

Proof. The proof of (4.11) follows by (2.5) and linearity of $\Delta_{\ell}^{-1}$. Now, (4.12) will be obtained by taking $m=1$ in (4.11) and using (4.6), (4.7), and (4.9).

Theorem 4.6. Let $\ell \in(0, \infty)$ and $n \in \mathbb{N}(1)$. Then,

$$
\lim _{\ell \rightarrow 0} \Gamma_{\ell}(n)=\lim _{\ell \rightarrow 0} \Gamma_{\ell}((n))=(n-1) !=\Gamma(n) .
$$

Proof. As $\ell \rightarrow 0$, all the terms except the last term of (4.12) will be zero. Also, since $\lim _{k \rightarrow \infty} \ell e^{-\ell} /\left(1-e^{-\ell}\right)=1$, by $(4.9)$, we arrive

$$
\lim _{\ell \rightarrow 0} \Gamma_{\ell}(n)=\lim _{\ell \rightarrow 0}(n-1) \Gamma_{\ell}((n+1))=\lim _{\ell \rightarrow 0} \Gamma_{\ell}((n)) .
$$

Now, (4.13) follows by replacing $n$ by $n-1$ and taking limit $\ell \rightarrow 0$ on (4.9).

Theorem 4.7. Let $k \in[\ell, \infty)$ and $j=k-[k / \ell] \ell$. Then,

$$
\sum_{r=1}^{[k / \ell]}\left(\frac{k}{\ell}-r\right) \Gamma\left(\frac{k}{\ell}-r+1\right)=\Gamma\left(\frac{k}{\ell}+1\right)-\Gamma\left(\frac{j}{\ell}+1\right)
$$

Proof. From (2.1) and (2.2), we have

$$
\Delta_{\ell} \Gamma\left(\frac{k}{\ell}+1\right)=\frac{k}{\ell} \Gamma\left(\frac{k}{\ell}+1\right)
$$

which yields

$$
\Delta_{\ell}^{-1}\left[\frac{k}{\ell} \Gamma\left(\frac{k}{\ell}+1\right)\right]_{j}^{k}=\Gamma\left(\frac{k}{\ell}+1\right)
$$

Now, the proof follows from (2.6) and (4.17). 
Theorem 4.8. Let $k \in[\ell, \infty)$ and $j=k-[k / \ell] \ell$. Then,

$$
\sum_{r=1}^{[k / \ell]} \frac{(k / \ell)-r}{(k / \ell)-r+1} \frac{1}{\Gamma((k / \ell)-r+1)}=\frac{1}{\Gamma((j / \ell)+1)}-\frac{1}{\Gamma((k / \ell)+1)}
$$

Proof. From (2.1) and (2.2), we find

$$
\Delta_{\ell}\left[\frac{1}{\Gamma((k / \ell)+1)}\right]=-\frac{(k / \ell)}{((k / \ell)+1) \Gamma((k / \ell)+1)},
$$

which yields by (2.2),

$$
-\Delta_{\ell}^{-1}\left[\frac{(k / \ell)}{((k / \ell)+1) \Gamma((k / \ell)+1)}\right]_{j}^{k}=\frac{1}{\Gamma((k / \ell)+1)}-\frac{1}{\Gamma((j / \ell)+1)}
$$

Now, the proof follows from (2.6) and (4.20).

Corollary 4.9. Let $k \in[\ell, \infty)$ and $j=k-[k / \ell] \ell$. Then,

$$
\sum_{r=0}^{\infty} \frac{(k / \ell)+r}{(k / \ell)+r+1} \frac{1}{\Gamma((k / \ell)+r+1)}=\frac{1}{\Gamma((k / \ell)+1)} .
$$

Proof. The proof follows from (4.1) and (4.20).

Corollary 4.10. (i) When $k=0$,

$$
\sum_{r=0}^{\infty} \frac{r}{r+1} \frac{1}{\Gamma(r+1)}=1
$$

(ii) When $k=1$ and $\ell=0.5$,

$$
\sum_{r=0}^{\infty} \frac{2+r}{3+r} \frac{1}{\Gamma(3+r)}=\frac{1}{\Gamma 3}=\frac{1}{2 !}
$$

(iii) When $k=n \ell, n \in N(1)$,

$$
\sum_{r=0}^{\infty} \frac{n+r}{n+1+r} \frac{1}{\Gamma(n+1+r)}=\frac{1}{\Gamma(n+1)}=\frac{1}{n !}
$$

\section{Acknowledgment}

This paper is supported by the University Grants Commission, SERO, Hyderabad, India. 


\section{References}

[1] K. S. Miller and B. Ross, An Introduction to the Fractional Calculus and Fractional Differential Equations, John Wiley \& Sons, New York, NY, USA, 1993.

[2] S. G. Samko, A. A. Kilbas, and O. I. Marichev, Fractional Integrals and Derivatives, Gordon and Breach Science Publishers, Yverdon, Switzerland, 1993.

[3] R. P. Agarwal, Difference Equations and Inequalities, vol. 228, Marcel Dekker, New York, NY, USA, 2nd edition, 2000.

[4] R. Almeida and D. F. M. Torres, "Necessary and sufficient conditions for the fractional calculus of variations with Caputo derivatives," Communications in Nonlinear Science and Numerical Simulation, vol. 16, no. 3, pp. 1490-1500, 2011.

[5] R. L. Magin, Fractional Calculus in Bioengineering, Begell House, 2006.

[6] A. B. Malinowska and D. F. M. Torres, "Generalized natural boundary conditions for fractional variational problems in terms of the Caputo derivative," Computers \& Mathematics with Applications, vol. 59, no. 9, pp. 3110-3116, 2010.

[7] J. Sabatier, O. P. Agrawal, and J. A. Tenreiro Machado, Advances in Fractional Calculus, Springer, Dordrecht, The Netherlands, 2007.

[8] K. S. Miller and B. Ross, "Fractional difference calculus," in Univalent Functions, Fractional Calculus, and Their Applications (Koriyama, 1988), pp. 139-152, Horwood, Chichester, UK, 1989.

[9] N. R. O. Bastos, R. A. C. Ferreira, and D. F. M. Torres, "Discrete-time fractional variational problems," Signal Processing, vol. 91, no. 3, pp. 513-524, 2011.

[10] R. A. C. Ferreira and D. F. M. Torres, "Fractional $h$-difference equations arising from the calculus of variations," Applicable Analysis and Discrete Mathematics, vol. 5, no. 1, pp. 110-121, 2011.

[11] G. S. F. Frederico and D. F. M. Torres, "A formulation of Noether's theorem for fractional problems of the calculus of variations," Journal of Mathematical Analysis and Applications, vol. 334, no. 2, pp. 834-846, 2007.

[12] M. M. Susai Manuel, G. B. A. Xavier, V. Chandrasekar, and R. Pugalarasu, “Theory and application of the generalized difference operator of the $n t h$ kind (Part I)," Demonstratio Mathematica, vol. 45, no. 1, pp. 95-106, 2012.

[13] M. M. S. Manuel, G. B. A. Xavier, and E. Thandapani, “Theory of generalised difference operator and its applications," Far East Journal of Mathematical Sciences (FJMS), vol. 20, no. 2, pp. 163-171, 2006. 


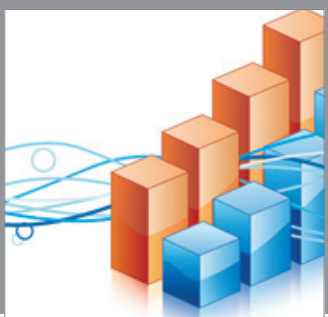

Advances in

Operations Research

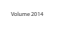

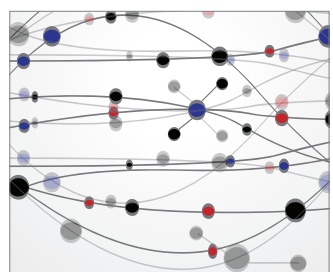

\section{The Scientific} World Journal
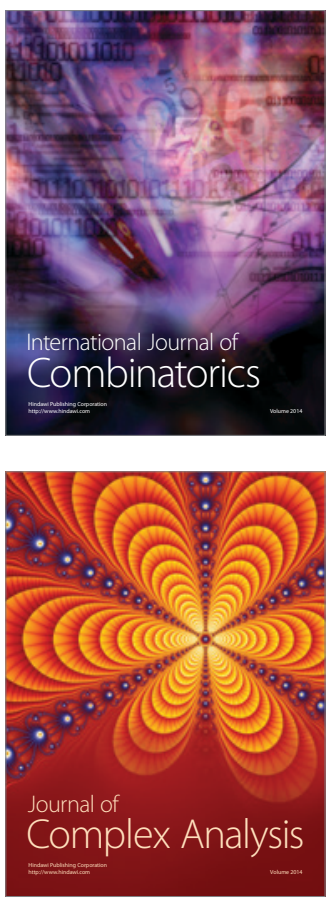

International Journal of

Mathematics and

Mathematical

Sciences
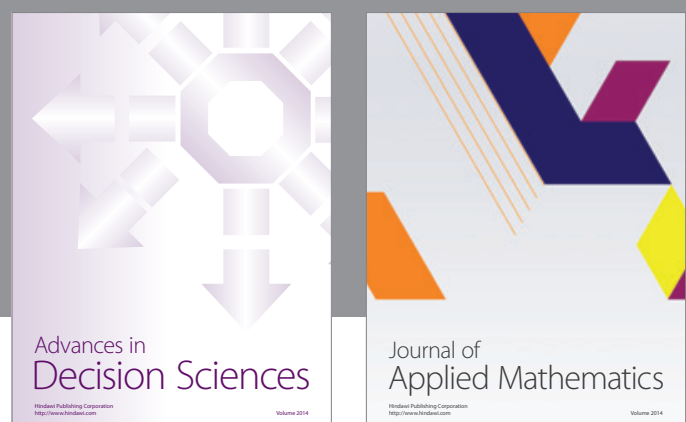

Journal of

Applied Mathematics
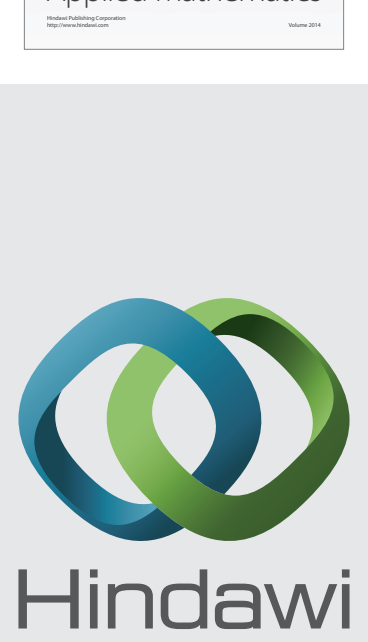

Submit your manuscripts at http://www.hindawi.com
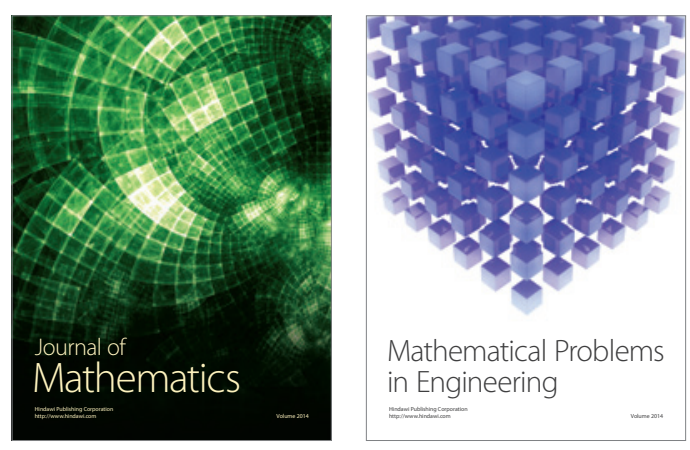

Mathematical Problems in Engineering
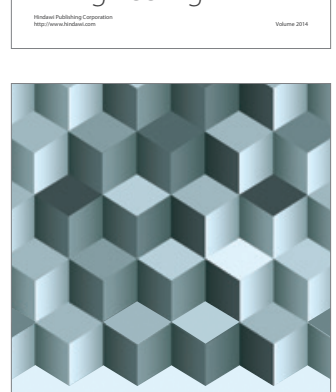

Journal of

Function Spaces
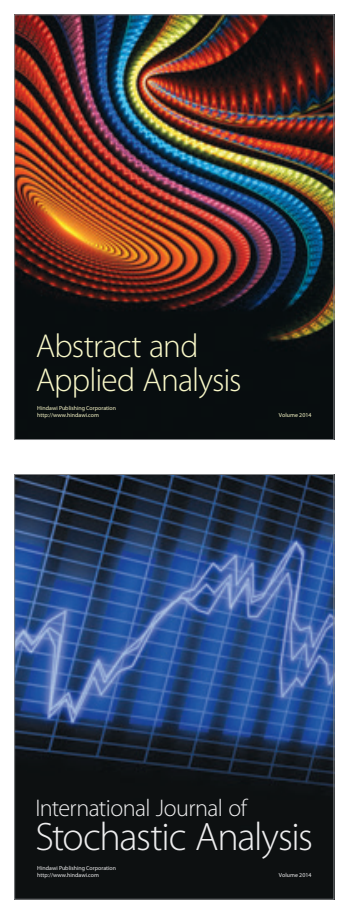

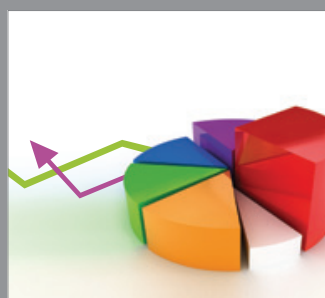

ournal of

Probability and Statistics

Promensencen
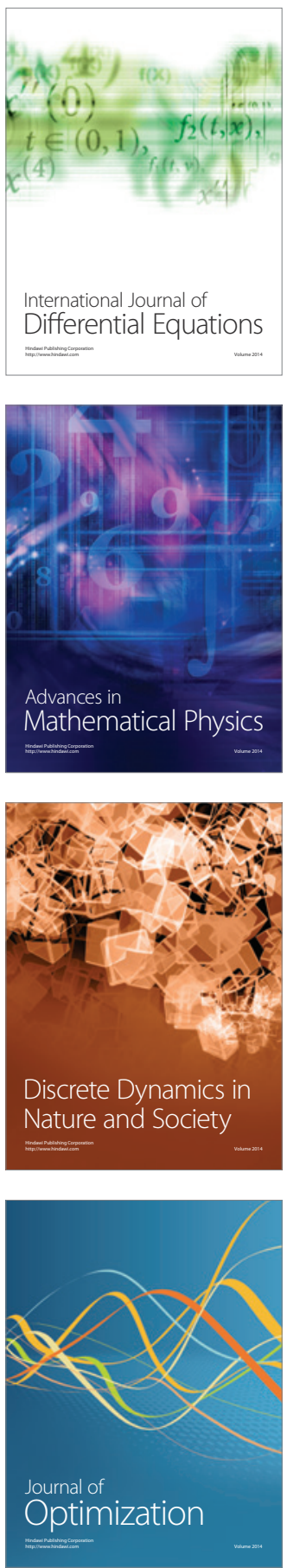